\title{
Síndrome da Apneia Obstrutiva do Sono: Avaliação da Ansiedade em Pacientes Recém-diagnosticados
}

\author{
Micheli Aparecida Gomes dos Santos* \\ Tatiana de Cássia Nakano* \\ Pontifícia Universidade Católica de Campinas, Campinas, SP, Brasil
}

\begin{abstract}
RESUMO
Com o objetivo de analisar e identificar os níveis de ansiedade em uma amostra de portadores da síndrome da apneia obstrutiva do sono, antes e após um mês de tratamento clínico ou cirúrgico, a Escala de Ansiedade Beck foi aplicada em uma amostra de 18 pacientes com esse diagnóstico. Resultados mostraram que 55,5\% dos participantes apresentavam ansiedade na primeira avaliação (antes do início do tratamento), entre os níveis leve, moderado e grave. Na segunda avaliação (um mês após início do tratamento) os pacientes apresentaram diminuição significativa na média de sintomas de ansiedade pós-início de tratamento $(\mathrm{z}=-3,51, \mathrm{p}<0,000)$. Estudos envolvendo outras variáveis, tais como adaptação ao tratamento, avaliação da qualidade do sono e efeitos de um programa de intervenção são recomendados.
\end{abstract}

Palavras-chave: avaliação psicológica; ansiedade; transtornos do sono.

\begin{abstract}
Obstructive Sleep Apnea Syndrome: Anxiety Assessment in Patients Newly Diagnosed

Aiming to analyze and identify the levels of anxiety in a sample of patients with obstructive sleep apnea syndrome before and after a month of medical or surgical treatment, the Beck Anxiety Scale was administered to a sample of 18 patients with this diagnosis. Results showed that $55.5 \%$ of the participants reported anxiety at the first assessment (before starting treatment), between the low, moderate and severe levels. In the second evaluation (one month after initiation of treatment) patients showed a significant decrease in mean symptoms of post-treatment initiation $(z=-3.51, p<0.000)$ anxiety. Studies involving other variables, such as adaptation to treatment, assessment of sleep quality and effects of a program of intervention are recommended.
\end{abstract}

Keywords: psychological assessment, anxiety, sleep disorders.

Na sociedade atual, algumas atividades são priorizadas em detrimento ao sono, ainda que ele se mostre imprescindível para a manutenção de uma vida saudável (Mathias, Sanchez, \& Andrade, 2006). A redução do tempo de dormir tornou-se um hábito comum na atualidade, guiado pelas exigências e oportunidades da sociedade moderna. Pesquisas mostram que, no decorrer de 40 anos, a duração autorreportada do sono diminuiu de 1,5 a 2 horas nos Estados Unidos. A proporção de jovens adultos que apresentam período de sono inferior a sete horas por noite aumentou de 15,6\% em 1960 para 37,1\%, em 2001-2002 (Crispim et al., 2007). Segundo a Comissão Americana de Pesquisa nos Distúrbios do Sono, os transtornos do sono atingem aproximadamente 40 milhões de pessoas nos Estados Unidos. Esses distúrbios podem causar sonolência diurna, diminuir a qualidade de vida e implicar risco para os pacientes, de tal forma que o diagnóstico preciso desses distúrbios torna-se fundamental do ponto de vista social e econômico (American Sleep Disorders Association, 1997).

De acordo com Cardoso et al. (2009), os transtornos do sono trazem consigo diversas repercussões, podendo-se citar, dentre elas, a perda da qualidade de

* Endereço para correspondência: Micheli Aparecida Gomes dos Santos - micheligomes@yahoo.com.br

* Endereço para correspondência: Tatiana de Cássia Nakano - tatiananakano@hotmail.com 
vida, disfunção autonômica, diminuição do desempenho profissional ou acadêmico, aumento na incidência de transtornos psiquiátricos e diminuição da vigilância, com prejuízos na segurança pessoal e consequente aumento do número de acidentes. As pessoas que dormem mal tendem a ter mais morbidades, menor expectativa de vida e envelhecimento precoce. No que se refere ao desempenho cognitivo, diversas são as alterações que podem relacionar-se de forma direta a prejuízos decorrentes de distúrbios do sono, como: dificuldades de fixar e manter atenção, prejuízo de memória e na capacidade de planejamento estratégico, prejuízo motor leve, principalmente relacinado à coordenação motora fina (agilidade e precisão), dificuldade de controlar impulsos e alteração de raciocínio (Bittencourt, Silva, Santos, Pires, \& Mello, 2005). Nesse mesmo sentido, Incalzi et al. (2004) destacam que o funcionamento mental é integrado, de maneira que, se existe prejuízo em alguma função, o desempenho em outra pode ser afetado em maior ou menor grau.

Gotsopoulos, Chen, Qian e Cistulli (2002) afirmam que a síndrome da apneia obstrutiva do sono (SAOS), dentre os distúrbios respiratórios do sono, é um dos mais contundentes, diminuindo substancialmente a saúde e qualidade de vida de seus portadores. Trata-se de uma doença crônica de cunho evolutivo. Caracteriza-se por repetidos episódios de obstrução parcial ou completa das vias aéreas superiores durante o sono, frequentemente associada à interrupção do mesmo e queda na saturação da oxi-hemoglobina (American Academy of Sleep Medicine Task Force, 1999).

Segundo Young et al. (1993), estima-se que, em adultos de países ocidentais, $4 \%$ dos homens e $2 \%$ das mulheres sejam acometidos pela SAOS de maneira clinicamente significante. Pode ocorrer em qualquer idade, mas acomete principalmente homens obesos entre 40 e 60 anos, sendo menos frequente em mulheres antes da menopausa. É mais prevalente no sexo masculino devido a diferenças anatômicas das vias aéreas superiores (VAS), perfil hormonal e distribuição adiposa do tipo central nos homens (tronco e pescoço). A prevalência tende a aumentar em faixas etárias avançadas (Mancini, Aloe, \& Tavares, 2000). A sintomatologia clínica que se observa nos pacientes com SAOS tem a particularidade de mostrar indícios que se manifestarão exclusivamente durante o sono (por exemplo, apneia e ronco) e outros, como a sono- lência, que aparecerão alterando o comportamento diurno (Noal, Menezes, Canani, \& Siqueira, 2008).

A fragmentação e a privação do sono secundárias à SAOS podem levar a alterações de personalidade, com surtos de ansiedade, acessos de depressão profunda, comportamento automático, cefaleias matinais recorrentes ou náusea matinal (Lowe et al., 1997). Tem sido observada uma melhora nos sintomas psicológicos após tratamento cirúrgico (Li et al., 2004) ou uso de CPAP, sigla em inglês de Continuous Positive Airway Pressure, ou seja, pressão positiva contínua na via aérea (Bardwell et al. 2007; Derderian, Bridenbaugh, \& Rajagopal, 1988; Schwartz \& Karatinos, 2007).

A SAOS é caracterizada por sintomas noturnos e diurnos. Nos sintomas noturnos, estão presentes as pausas respiratórias, sono agitado com múltiplos despertares, noctúria, sudorese, engasgos, insônia noturna. Dentre os diurnos estão: hipersonolência diurna, cefaleia matinal, déficits de memória e atenção, alterações de personalidade, redução da libido, sintomas depressivos (principalmente nas mulheres), ansiedade, hipertensão pulmonar, problemas sexuais e estresse oxidativo (Mancini, 2000; Peppard, Young, Palta, \& Skatrud, 2000; Stepanski, 2002). Como consequência, diversos outros quadros têm se mostrado associados, conforme pode ser confirmado em estudo realizado em 2005 com 4060 americanos, no qual verificou-se que, nos 21,8\% diagnosticados com SAOS, 11,9\% foram diagnosticados com depressão, $16,7 \%$ com ansiedade elevada, 5,1\% com psicose e 3,3\% com transtorno bipolar (Glebocka et al., 2006).

Diante da importância de se investigar a relação entre tais diagnósticos e a SAOS, o presente estudo buscou enfocar a ansiedade associada a esse distúrbio do sono, dado o fato de que os transtornos de ansiedade são frequentes e incapacitantes. Segundo Dalgalarrondo (2000), a ansiedade é definida como um estado de humor desconfortável, uma apreensão negativa em relação ao futuro, uma inquietação interna desagradável. Inclui manifestações somáticas e fisiológicas (dispneia, taquicardia, vasoconstrição ou dilatação, tensão muscular, parestesias, tremores, sudorese, tontura, entre outros) e manifestações psíquicas (inquietação interna, apreensão, desconforto mental, entre outros), salientadas por Savoia (2010). De acordo com Sadock e Sadock (2007), tal quadro exerce efeito sobre o pensamento, percepção e aprendizado, tende a 
produzir confusão e distorções perceptivas, podendo interferir no aprendizado, reduzindo a concentração, a memória e a capacidade de associação.

No que diz respeito ao aspecto psicológico, diversos estudos investigando a relação entre SAOS e ansiedade são encontrados na literatura internacional (Sahbaz et al., 2008; Sanchez, Buela-Casal, Bermudez, \& Casas-Maldonado, 2001; Yue et al., 2003). Os resultados mais comuns demonstram que os doentes com SAOS apresentam níveis elevados de ansiedade, que se traduzem na irritabilidade e tensão, que surgem como consequência direta da privação do sono e diminuição da energia (Bardwell et al., 1999). Esse quadro evidencia uma urgente necessidade de compreender melhor o papel da ansiedade na SAOS (Andrews, Tian, \& Oei, 2004).

Em resumo, a literatura científica tem demonstrado que o prejuízo do descanso noturno, causado pelas fragmentações do sono em pessoas acometidas pela Síndrome da Apneia Obstrutiva do Sono, pode refletir-se emocionalmente, gerando, além de sintomas físicos tais como sonolência e cansaço no dia seguinte, também sintomas psíquicos, como a ansiedade. Diante dessa constatação, o objetivo do presente estu- do foi comparar sintomas de ansiedade em portadores de SAOS, moderada e grave, antes e após um mês do início do tratamento clínico ou cirúrgico.

\section{MÉTODO}

\section{Participantes}

A amostra foi composta por 18 pacientes, com idade média de 51,83 anos, mínima de 26 anos e máxima de 74 anos $(\mathrm{DP}=13,46)$. Destes, 72,2\% $(\mathrm{n}=13)$ eram do sexo masculino. No que se refere à escolaridade, houve maior frequência de participantes com ensino fundamental incompleto $(55,6 \% \mathrm{n}=10)$ e em relação ao estado civil 83,3\% ( $n=15)$ eram casados. Quanto a ocupação profissional dos participantes, $16,7 \%$ estavam aposentados no momento da pesquisa, sendo que, em relação ao restante da amostra, diferentes ocupações foram encontradas (auxiliar de enfermagem, porteiro, auxiliar administrativo, protético dentário, zelador, cuidador de idosos, eletricista, professora, motorista, militar, técnico de informática, comerciante, ajudante geral e empresário), cujos dados podem ser visualizados na Tabela 1 .

Tabela 1

Distribuição da Amostra a Partir dos Dados Sociodemográficos

\section{Distribuição dos participantes}

$\%$

Masculino

Feminino

26 a 40

41 a 59

$\geq 60$

Casado

Estado Civil

Solteiro

Divorciado

Ensino Fundamental Incompleto

Ensino Médio Completo

Ensino Fundamental Completo

Ensino Superior Completo

Ensino Superior Incompleto n

13

5

4

9

5

15

2

1

10

4

2

1

1
72,2

27,8

22,2

50

27,8

83,3

11,1

5,6

55,6

22,2

11,1

5,6

5,6 
Outros tipos de dados foram coletados, sendo o índice de massa corporal (IMC), antecedentes psicológicos e/ou psiquiátricos e avaliação quanto ao tipo de SAOS. Em relação ao primeiro dado, 33,3\% apresentavam sobrepeso, $27,8 \%$ estavam com obesidade grau I, $16,7 \%$ encontravam-se com obesidade grau III e somente 5,6\% estava com peso dentro da faixa de normalidade. Dos participantes, $72,2 \%$ nunca fizeram acompanhamento psicológico e $27,8 \%$ já haviam feito em momento anterior. Somente 5,6\% estava fazendo algum tipo no momento da avaliação. No que se refere ao tipo de SAOS, este foi determinado por meio de polissonografia de noite inteira e verificou-se que $72,2 \%(n=13)$ da amostra sofria de SAOS grave e $27,8 \%(n=5)$ SAOS moderada. Pacientes que sofriam de SAOS leve não foram encontrados no exame.

O estudo foi realizado em um Ambulatório especializado em Distúrbios Respiratórios Obstrutivos do Sono (DROS), pertencente ao Serviço de otorrinolaringologia de um hospital universitário, do interior do estado de São Paulo.

\section{Materiais e Instrumentos}

A coleta de dados fez uso de um questionário de identificação elaborado com a finalidade de se obter dados sociodemográficos dos participantes e o Inventário de Ansiedade Beck (BAI) (Cunha, 2001).

O BAI é um instrumento de autorrelato, que avalia a intensidade dos sintomas de ansiedade, sendo constituído por 21 itens, que apresentam afirmações descritivas de sintomas de ansiedade a serem respondidas pelo sujeito com referência a si mesmo, numa escala de 4 pontos, que refletem níveis de gravidade crescente de cada sintoma, a saber: 1) absolutamente não; 2) levemente: não me incomodou muito; 3) moderadamente: foi muito desagradável, mas pude suportar; 4) gravemente: dificilmente pude suportar. O escore total no teste é resultado da soma das pontuações obtidas em caa um dos itens, podendo variar de 0 a 63 , de modo a permitir a classificação da ansiedade em níveis de intensidade (mínimo, leve, moderado e grave). O tempo para seu preenchimento varia entre 5 a 10 minutos.
As propriedades psicométricas da escala foram estudadas, a princípio, baseadas em dados colhidos em pacientes psiquiátricos. Para os estudos iniciais da BAI uma amostra de 160 pacientes ambulatoriais com diagnóstico misto foi utilizada. Posteriormente, foi selecionada uma amostra de pacientes ambulatoriais, com diagnósticos de transtorno de ansiedade. De acordo com os autores, a BAI compreende itens que são representativos de ansiedade ao mesmo tempo em que evita incluir sintomas compartilhados com quadros depressivos. Com a versão brasileira, desenvolveu-se estudos para estimar a correlação entre a BAI e o IDATE (Spielberger, Biaggio, \& Natalício, 1979), constatando-se que apresenta relação significante com A-Traço $(r=0,78 ; p<0,001)$ e com A-Estado $(r=0,76$; $\mathrm{p}<0,001)$.

\section{Procedimentos}

O presente projeto foi aprovado para execução pelo Comitê de Ética em Pesquisa de universidade que o abriga. Os pacientes assinaram um Termo de Consentimento Livre e Esclarecido no qual concordavam em participar da pesquisa e, por meio do mesmo, permitindo que os dados fossem divulgados para fins científicos, anonimamente. Salienta-se que, em nenhum momento, o oferecimento do atendimento médico ou tratamento foi condicionado à participação na pesquisa.

$\mathrm{Na}$ primeira sessão com o participante, antes do início do tratamento para SAOS, foi aplicado o questionário sociodemográfico e a escala de ansiedade. No segundo encontro, após um mês de tratamento (clínico ou cirúrgico), somente a escala.

\section{RESULTADOS}

A avaliação dos resultados obtidos no BAI permite a identificação dos níveis de ansiedade apresentados pelos indivíduos e revelou que, a maior parte dos participantes $(44,4 \%)$, apresentou grau mínimo de sintomas de ansiedade na primeira avaliação, considerado normal, sendo menos frequente o grau grave $(11,1 \%$ dos casos). Os resultados podem ser visualizados na Figura 1. 


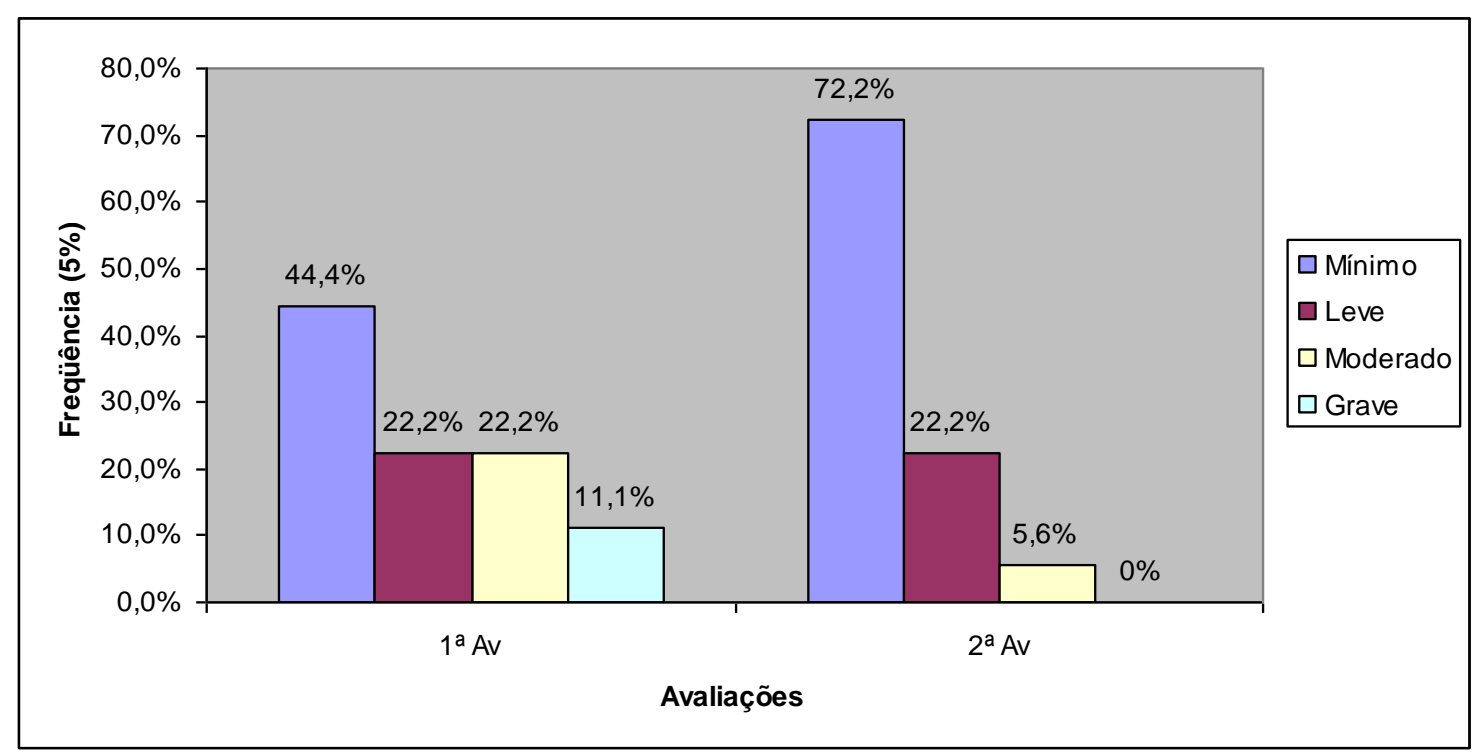

Figura 1. Porcentagem dos níveis de ansiedade pré e pós-intervenção.

Mudança importante pode ser notada na segunda avaliação, dado o fato de que, não aparecem mais os casos graves, havendo também diminuição dos casos moderados (de 22,2\% para 5,6\%). Como consequência há o aumento de pacientes classificados com ansiedade mínima.
A fim de melhor visualizar os dados, a estatística descritiva do pré e pós-tratamento são apresentadas na Tabela 2. Nela pode-se verificar que a média de sintomas de ansiedade cai bastante da primeira para a segunda avaliação $(M=11,83$ e $M=4,50$, respectivamente).

\section{Tabela 2}

Estatística Descritiva para Ansiedade Pré e Pós-tratamento

\begin{tabular}{cccccccc} 
& N & Média & Mediana & Mín. & Máx. & DP & EP \\
1a Avaliação & 18 & 11,83 & 11,0 & 1 & 40 & 11,04 & 2,60 \\
2a Avaliação & 18 & 4,50 & 2,0 & 0 & 19 & 6,08 & 1,43 \\
\hline
\end{tabular}

A fim de detectar se as diferenças entre a média de sintomas na BAI entre os sujeitos, antes e após iniciado o tratamento eram significativas, o teste não paramétrico de Wilcoxon foi selecionado. Seu resultado apontou que os pacientes apresentaram uma diminuição significativa na média de sintomas de ansiedade pós-tratamento $(\mathrm{z}=-3,51, \mathrm{p}<0,000)$. Desse modo podese visualizar que os pacientes demonstraram melhora significativa nos sintomas na segunda avaliação, de modo a se poder afirmar, para esse grupo de indivíduos, que o simples início do tratamento, mesmo após um curto período, teve o efeito desejado.

Os dados obtidos com o uso do BAI também foram investigados, de maneira mais detalhada e qualitativa, visando-se identificar os sintomas de ansiedade mais frequentes nesta amostra, em ambas as avaliações. Os resultados demonstraram que o nervosismo é o sintoma mais comumente relatado pelos pacientes, persistindo na segunda avaliação (em $66,7 \%$ dos casos na primeira avaliação e $27,8 \%$ na segunda avaliação). Bastante comum também é a tontura e a dificuldade para respirar (presente em 44,4\% dos casos) antes do início do tratamento e indigestão ou desconforto no abdômen $(27,8 \%)$ após um mês de tratamento.

Uma segunda análise, visando comparar a ansiedade entre o grupo de apneicos moderados $(n=13)$ com o grupo de apneicos graves $(n=5)$, nos dois momentos avaliados foi realizada, em caráter exploratório, dada 
a desigualdade no número de participantes em cada grupo. Os resultados apontaram para uma média menor de sintomas presente no grupo com SAOS moderada $(\mathrm{M}=7,0, \mathrm{DP}=8,15)$, quando comparado ao grupo com SAOS grave $(\mathrm{M}=13,69, \mathrm{DP}=11,70)$ na primeira avaliação, pré-tratamento. Na segunda avaliação, pós início do tratamento, a média de sintomas diminui para ambos os grupos $(\mathrm{M}=0,80, \mathrm{DP}=1,30$ para moderada e $M=5,92, D P=6,62$ para grave), ainda que a diminuição no grupo com SAOS grave tenha sido maior.

Com o objetivo de verificar se essas diferenças relatadas eram significativas, novamente o teste não paramétrico de Mann-Whitney foi empregado, visto que o mesmo possibilita o teste de diferenças entre duas condições e diferentes participantes (Field, 2009). Os resultados demonstraram que não houve diferença significativa intergrupo (moderada $x$ grave) na primeira avaliação $(U=19,5, \quad z=-1,29, \quad p=0,197)$, assim como na avaliação realizada após iniciado o tratamento $(\mathrm{U}=17, \mathrm{z}=-1,57, \mathrm{p}=0,114)$.

Uma segunda análise, a partir do teste não paramétrico de Wilcoxon, foi utilizada na comparação intragrupo dos escores provenientes dos mesmos participantes (SAOS moderada pré e pós-tratamento; SAOS grave pré e pós-tratamento), cujos resultados apontaram que os pacientes com SAOS moderada apresentaram uma diminuição significativa na média de sintomas de ansiedade pós-tratamento $(\mathrm{z}=-2,032$, $\mathrm{p}<0,042$ ), o mesmo ocorrendo em relação aos pacientes com SAOS grave $(\mathrm{z}=-2,944, \mathrm{p}<0,003)$, de modo a demonstrar que o tratamento foi eficaz na melhora dos sintomas de ansiedade para os dois níveis de gravidade da doença.

Por fim, outra análise exploratória visou comparar os pacientes em função do tratamento (CPAP ou tratamento cirúrgico). Os resultados apontaram para uma diminuição importante nos sintomas de ansiedade no grupo que realizou tratamento com CPAP $(\mathrm{M}=12,73$, $\mathrm{DP}=11,32$ antes do tratamento e $\mathrm{M}=4,40, \mathrm{DP}=5,84$ após início do tratamento), sendo que, no grupo cirúrgico, tal diminuição não se mostrou tão importante $(\mathrm{M}=7,33, \mathrm{DP}=10,11$ antes do tratamento e $\mathrm{M}=5,00$ $\mathrm{DP}=8,66$ após início do tratamento).

Dadas as aparentes diferenças de médias, o teste não paramétrico de Wilcoxon foi aplicado e apontou, em relação aos pacientes que fizeram o tratamento com CPAP, a existência de diferença significativa nos sintomas de ansiedade da primeira para a segunda avaliação ( $\mathrm{z}=-3,17, \mathrm{p}=0,001)$, o mesmo não ocorrendo em relação ao grupo que passou por cirurgia $(z=-$ $1,60, p=0,109)$. De tal modo, foi possível verificar que o tratamento com CPAP mostrou-se mais eficaz para a redução dos sintomas de stress e ansiedade do que o tratamento cirúrgico, nos pacientes envolvidos na presente pesquisa.

\section{DISCUSSÃO}

Tomando-se o objetivo principal do estudo, de avaliar sintomas de ansiedade de pacientes recémdiagnosticados com SAOS, antes e após um mês de tratamento clínico ou cirúrgico, verificou-se que a presença desses sintomas se mostrou pouco incidente no grupo estudado, visto que a maior parte da amostra apresentou, antes do tratamento, nível mínimo de ansiedade, ainda que alguns casos de ansiedade moderada e grave tenham sido encontrados. A constatação de prevalência de nível baixo de ansiedade na amostra estudada vai de encontro com alguns resultados de pesquisas encontradas na literatura científica (Sahbaz et al., 2008; Sanchez, Buela-Casal, Bermudez, \& Casas-Maldonado, 2001; Yue et al., 2003), segundo os quais, é comum esse tipo de paciente apresentar níveis elevados de ansiedade (Bardwell et al., 1999).

Entretanto, antes de fazer comparações, convém salientar a diferença entre os estudos relatados e a pesquisa aqui apresentada. Esta última teve como foco a utilização de um instrumento psicológico para avaliação da ansiedade em pacientes com SAOS, sendo este um dos seus diferenciais, dado o fato de que grande parte dos estudos encontrados na literatura ampararam-se na investigação da ansiedade e seus efeitos físicos e/ou orgânicos. Como exemplo podemos citar o estudo conduzido por Kumar et al. (2009), o qual teve como objetivo determinar se apneicos expressando sintomas de ansiedade apresentariam lesões em regiões específicas do cérebro. Ainda que o BAI também tenha sido utilizado na pesquisa citada, por meio de ressonância magnética foram avaliados 46 apneicos e 66 sujeitos controle, os autores focaram a existência de lesões em áreas do cérebro que regulam a emoção, comumente presentes no grupo apneico.

Ainda que, antes de iniciado o tratamento, a maioria dos pacientes já não apresentava sintomas característicos de ansiedade, apresentando sintomas no grau considerado mínimo, pode-se verificar que, após ini- 
ciado o tratamento o índice de pacientes que apresentavam sintomas de ansiedade diminuiu significativamente de modo a se poder supor a eficácia do tratamento com CPAP sobre a ansiedade dos pacientes. Resultados diferentes foram apresentados por Carissimi (2011) ao investigar o efeito da hipóxia, sonolência e sono profundo sobre a resposta de sintomas psicológicos após o tratamento com o CPAP em 73 pacientes com apneia grave que responderam ao Symptom Checklist-90 (SCL-90). Os resultados apontaram que os pacientes tratados com CPAP não apresentaram melhora significativa nas dimensões de ansiedade, depressão e ansiedade fóbica. Essa diferença merece ser melhor investigada futuramente dada a possibilidade de que outros fatores não considerados e/ou controlados em ambos os estudos possam ter inferido nos resultados, podendo-se citar, dentre eles, inclusive as diferentes medidas utilizadas para avaliação do construto.

Ainda referente aos sintomas de ansiedade, na primeira avaliação, importante parte dos avaliados revelaram sentir incapacidade de relaxar, medo que aconteça o pior e medo de perder o controle. Embora estes não tenham sido os sintomas mais prevalentes, sua importância refere-se ao fato de que, ao serem questionados sobre os mesmos, os pacientes mostraram-se apreensivos em relação ao recente diagnóstico e ao tratamento. Tal preocupação também foi relatada no estudo conduzido por Yue et al. (2003) com o objetivo de avaliar a relação entre a SAOS e problemas psicológicos, cujos resultados apontaram escores significativamente mais elevados entre os sujeitos apneicos do que entre os do grupo controle com relação à somatização, ansiedade, hostilidade e depressão. Do mesmo modo, escores mais elevados na escala de sonolência foram apresentados entre os apneicos do que entre os sujeitos controle, fato explicado pela fragmentação do sono ocorrido entre os primeiros.

$\mathrm{Na}$ amostra estudada, quando comparados os grupos de apneicos moderados e graves, os resultados apontam para a existência de diferenças estatisticamente significantes com relação aos níveis de ansiedade após o tratamento. Dessa maneira foi possível verificar que o tratamento foi eficaz na melhora dos sintomas para os dois níveis da doença (moderado e grave). Pesquisa com resultados semelhantes foi desenvolvida por Franco (2009), cujo objetivo geral foi avaliar a correlação entre a gravidade da SAOS, marcadores de estresse oxidativo e presença de sintomas depressivos e ansiosos. Os participantes foram 38 adultos, do sexo masculino, com diagnóstico de SAOS por polissonografia de noite inteira, com idade entre 18 e 60 anos, avaliados para nível de sonolência subjetiva por meio da escala de Epworth e para sintomas depressivos e ansiosos pelas escalas de Hamilton depressão (HAM-D) e ansiedade (HAM-A). Os resultados demonstraram um aumento progressivo e significativo no escore total de sintomas depressivos $(\mathrm{p}=0,001)$ e no escore geral dos sintomas ansiosos $(\mathrm{p}=0,004)$ diretamente proporcional à gravidade da apneia, comparando os grupos leve e grave. Concluiuse que quanto maior a gravidade da SAOS, maior a fragmentação do sono e maior é a incidência de sintomas diurnos, principalmente sonolência, sintomas depressivos e ansiosos. Diferentemente do aqui relatado (tanto na primeira avaliação quanto na segunda não foram encontradas diferenças significativas em relação ao nível de ansiedade dentre os grupos moderado e grave), naquele conduzido por Franco (2009) tais sintomas mostraram-se mais intensos e presentes na apneia grave. Convém salientar, entretanto, que o estudo citado comparou diferentes grupos (leve e grave, ao passo que aqui foram comparados pacientes moderados e graves), de modo que a diferença entre eles talvez não se faça tão discriminativa.

Ao se comparar a presença de ansiedade em relação ao tratamento utilizado verificou-se que os pacientes apresentaram uma redução significativa na sintomatologia após o tratamento com o CPAP, dado semelhante ao encontrado por Sanchez, Buela-Casal, Bermudez e Casas-Maldonado (2001), os quais verificaram, em uma pesquisa com 51 pacientes que passaram por tratamento com o CPAP, que os resultados mostraram diferenças estatisticamente significativas nos níveis de ansiedade-traço após 1 mês e após 3 meses de tratamento $(\mathrm{p}<0,05)$ e em estados de ansiedade, após 3 meses de tratamento $(p<0,01)$, de maneira que as mudanças, nos pacientes que fizeram uso desse tipo de tratamento, mostraram-se duradouras.

No entanto, os pacientes do presente estudo que passaram por tratamento cirúrgico denominado faringoplastia lateral (FL), não apresentaram uma melhora significativa na sintomatologia de ansiedade, o que não corresponde a alguns dados encontrados na literatura. Li et al. (2004), por exemplo, investigaram se os sintomas psicológicos apresentados por apneicos poderiam ser aliviados após seis meses de uma intervenção cirúrgica. A escala 5 Item de Saúde Mental (MH5) foi utilizada para avaliar as alterações de humor pré 
e pós-operatório em 84 pacientes. Este estudo demonstrou que pacientes com SAOS apresentaram, de uma forma geral, maior nível de ansiedade, depressão e mudanças de personalidade do que na população em geral. Desse modo os autores concluíram que a cirurgia pode melhorar significativamente o estado de humor entre os pacientes com SAOS, ainda que o efeito da cirurgia tenha sido leve, mas clinicamente relevante. Convém ressaltar, entretanto, que, devido ao pequeno intervalo de tempo entre as avaliações, os resultados podem ter sofrido influência de outros fatores, podendo-se citar, como exemplo, a necessidade de nova adaptação, por parte dos pacientes, resultante do uso do CPAP quanto em relação aos incômodos provenientes da cirurgia, tais como processo de cicatrização e efeitos colaterais de medicações.

De acordo com os dados encontrados, considerando-se a comparação entre o tipo de tratamento, foi possível verificar que após um mês de iniciada a intervenção médica, que o tratamento com CPAP mostrou-se mais eficaz para a redução dos sintomas de ansiedade do que o tratamento cirúrgico, nos pacientes envolvidos na presente pesquisa. No entanto, de acordo com Cahali, Formigoni, Gebrim e Miziara (2004), há de se considerar que o processo de cicatrização e retração da ferida cirúrgica ocorrem a médio e longo prazos. Ademais, nos primeiros dias de pós-operatório existe um edema da região faríngea, que pode estreitar a VAS, e piorar o quadro de SAOS neste período. Os autores afirmam que os resultados da FL são máximos após o período de três a seis meses do procedimento, inclusive apresentando melhores resultados clínicos e polissonográficos que a uvulopalatofaringoplastia (UPFP) (Cahali, 2003; Cahali, Formigoni, Gebrim, \& Miziara, 2004). Nesse sentido uma nova avaliação, após intervalo de tempo maior, é recomendada a fim de verificar se os dados obtidos se mantêm.

Os dados encontrados neste estudo também podem ser justificados pelas argumentações trazidas em alguns estudos, os quais sugerem que o sucesso do tratamento muitas vezes requer mudanças no modo de vida do paciente, como a redução do peso corporal, a suspensão de medicamentos benzodiazepínicos e a abstenção de bebida alcóolica (Levy, Pétin, Mayer, Wuyan, \& Veale, 1996; Man, 1996), as quais, muitas vezes, comprometem a adesão e os resultados nesses casos. Tal constatação ainda nos remete à uma ressalva a ser feita, dado o fato de que variações na prevalência de ansiedade usualmente mostram-se afetadas por características do paciente e métodos da avaliação, conforme apontado por Saunamäki e Jehkonen (2007). Segundo Margis, Picon, Cosner e Silveira (2003), a capacidade individual de interpretar, avaliar e elaborar estratégias de enfrentamento seriam, nesse sentido, provavelmente geneticamente influenciadas.

\section{CONSIDERAÇÕES FINAIS}

Ao se analisar e identificar os níveis de ansiedade em uma amostra de portadores da síndrome da apneia obstrutiva do sono, foi possível verificar a existência prévia de sintomas mínimos de ansiedade na amostra, antes do início do tratamento (clínico ou cirúrgico). Entretanto, uma diminuição significativa nos sintomas de ansiedade pós tratamento foi notada, de modo a justificar a importância desse procedimento para a melhoria da qualidade de vida desses pacientes.

A relevância científica desta pesquisa diz respeito à busca da compreensão do que ocorre emocionalmente nos pacientes acometidos pela SAOS. É importante ressaltar que este estudo foi realizado com uma amostra reduzida e que, portanto, as considerações aqui apresentadas devem ser consideradas de maneira cautelosa, evitando-se propagações antecipadas. Convém salientar que o reduzido número de participantes se justifica perante a limitação de tempo para a coleta de dados dado o caráter da pesquisa (dissertação de mestrado com prazos restritos, ainda que a pesquisadora tenha permanecido no local de coleta de dados durante o período de 6 meses) e a dificuldade em encontrar, no ambulatório em questão, pacientes que ainda não tivessem iniciado tratamento.

Sugere-se a realização de outros estudos, com uma amostra mais representativa de modo a se obter dados mais seguros. Levanta-se também a hipótese de que o recebimento de um diagnóstico, assim como a possibilidade de receber um tratamento, podem ter influenciado na redução da ansiedade dos pacientes, de modo que outros fatores, além do tratamento em si, podem ter contribuído para a redução dos sintomas. Nesse sentido outras variáveis merecem ser melhor investigadas em estudos futuros, tais como a adaptação ao tratamento, uso de um instrumento para avaliação da qualidade do sono ou ainda o oferecimento de um programa de intervenção, visando o controle da ansiedade e melhora da qualidade de vida desses pacientes.

Ademais, este trabalho apresentou limitações quanto à ausência do controle de variáveis como obesidade 
e uso de medicamentos, que poderiam interferir nos resultados, constituindo-se como variáveis intervenientes, e que merecem uma investigação em estudos posteriores.

\section{REFERÊNCIAS}

American Academy of Sleep Medicine Task Force (1999). Sleep-related breathing disorders in adults: recommendations for syndrome definition and measurement techniques in clinical research. Sleep, 22(5), 667-689.

American Sleep Disorders Association Report (1997). Practice parameters for the indications for polysomnography and related procedures. Sleep, 20, 406-422.

Bardwell, W. A., Norman, D., Ancoli-Israel, S., Loredo, J. S., Lowery, A., Lim, W., \& Dimsdale, J. E. (2007). Effects of 2-week nocturnal oxygen supplementation and continuous positive airway pressure treatment on psychological symptoms in patients with obstructive sleep apnea: a randomized placebo-controlled study. Behavioral Sleep Medicine, 5(1), 21-38.

Bittencourt, L. R. A., Silva, R. S., Santos, R. F., Pires, M. L. N., \& Mello, M. T. (2005). Sonolência excessiva. Revista Brasileira de Psiquiatria, 27 (Supl. I), 16-21.

Cahali, M.B. (2003). Lateral pharyngoplasty: a new treatment for obstructive sleep apnea hypopnea syndrome. Laryngoscope, 113(11), 1961-1968.

Cahali, M. B., Formigoni, G. G. S., Gebrim, E. M. M. S., Miziara, I. D. (2004). Lateral pharyngoplasty versus uvulopalatopharyngoplasty: a clinical, polysomnographic and computed tomography measurement comparison. Sleep, 27(5), 942-950.

Cardoso, H. C., Bueno, F. C. C., Mata, J. C., Alves, A. P. R., Jochims, I., Vaz Filho, I. H. R., \& Hanna, M. M. (2009). Avaliação da qualidade do sono em estudantes de medicina. Revista Brasileira de Educação Médica, 33(3), 349-355.

Carissimi, A. (2011). Examinando fatores causais de sintomas psicológicos através do SCL-90-R em pacientes com apneia do sono grave. (Dissertação de Mestrado). Universidade Federal do Rio Grande do Sul, Porto Alegre, RS.

Crispim, C. A., Zalcman, I., Dáttilo, M., Padilha, H. G., Tufik, S., \& Mello, M. T. (2007). Relação entre sono e obesidade: uma revisão da literatura. Arquivos Brasileiros de Endocrinologia \& Metabologia, 51(7), 10411049.

Cunha, J.A. (2001). Manual da versão em português das Escalas Beck. São Paulo: Casa do Psicólogo.

Dalgalarrondo, P. (2000). Psicopatologia e semiologia dos transtornos mentais. Porto Alegre: Artmed.

Derderian, S. S., Bridenbaugh, R. H., \& Rajagopal, K. R. (1988). Neuropsychologic symptoms in obstructive sleep apnea improve after treatment with nasal continuous positive airway pressure. Chest, 94(5), 1023-1027.

Field, A. (2009). Descobrindo a estatística usando o SPSS. Porto Alegre: Artmed.

Glebocka A., Kossowska A., \& Bednarek M. (2006). Obstructive sleep apnea and the quality of life. Journal of Physiology and Pharmacology, 57, (Supp. 4), 111-117.

Gotsopoulos, H. Chen, C. Qian, J., \& Cistulli, P. A. (2002). Oral appliance therapy improves symptoms in obstructive sleep apnea: a randomized, controlled trial. American Journal of Respiratory and Critical Care Medicine, 166 (5), 743-748.

Incalzi, R. A., Marra, C., Salvigni, B. L., Petrone, A., Gemma, A., Selvaggio, D., \& Mormile, F. (2004). Does cognitive dysfunction conform to a distinctive pattern in obstructive sleep apnea syndrome? Journal of Sleep Research, 13(1), 79-86.

Kendler, K. S., Neale, M. C., Kessler, R. C., Heath, A. C. \& Eaves, L. J. (1992). Generalized anxiety disorder in women. Archives of General Psychiatry, 49(4), 267-272.

Kendler, K. S., Walters, E. E., Neale, M. C., Kessler, R. C., Heath, A. C. \& Eaves, L. J. (1995). The structure of the genectic and environmental risk factors for six major psychiatric disorders in women. Archives of General Psychiatry, 52(5), 374-383.

Kessler, R. C., McGonagle, K. A., Zhao, S., Nelson, C. B., Hughes, M., Eshleman, S., Wittchen, H. U. \& Kendler, K. S. (1994). Lifetime and 12-month prevalence of DSM-III-R psychiatric disorders in the United States. Archives of General Psychiatry, 51(1), 8-19.

Kessler, R. C., Berglund, P., Demler, O., Merikangas, K. R. $\&$ Walters, E. E. (2005). Lifetime prevalence and age-ofonset distributions of DSM-IV disorders in the national comorbidity survey replication. Archives of General Psychiatry, 62(6), 593-602.

Kessler, R. C., Chiu, W. T., Demler, O., Merikangas, K. R. \& Walters, E. E. (2005). Prevalence, severity, and comorbidity of 12-month DSM-IV disorders in the national comorbidity survey replication. Archives of General Psychiatry, 62(6), 617-627.

Kumar, R., Macey, P.M., Cross, R. L., Woo, M.A., YanGo, F. L. \& Harper, R. M. (2009). Neural alterations associated with anxiety symptoms in obstructive sleep apnea syndrome. Depression and Anxiety, 26(5), 480-491.

Levy, P., Pétin, J. L., Mayer, P., Wuyam, B. \& Veale, D. (1996). Management of simples snoring, upper airway resistance syndrome and moderate sleep apnea syndrome. Sleep, 19(suppl.9), S101-S110.

Li, H. Y., Huang, Y. S., Chen, N. H., Fang, T. J., Liu, C. Y., \& Wang, P.C. (2004). Mood improvement after surgery for obstructive sleep apnea. Laryngoscope, 114(6), 1098-1102. 
Lowe, A. A., Özbek, M. M., Miyamoto, K., Pae E. K., \& Fleetham, J. A. (1997). Cephalometric and demographic characteristics of obstructive sleep apnea: an evaluation with partial least squares analysis. Angle Orthod, 67(2), 143-54.

Macey, P. M., Woo, M. A., Kumar, R., Cross, R. L., \& Harper, R. M. (2010). Relationship between obstructive sleep apnea severity and sleep, depression and anxiety symptoms in newly-diagnosed patients. Plos one 5(4), 16.

Man, G. C. W. (1996). Obstructive sleep apnea - diagnosis and treatment. Medical Clinics of North America, 80(4), 803-821.

Mancini, M. C., Aloe, F., \& Tavares, S. (2000). Apneia do sono em obesos. Arquivos Brasileiros de Endocrinologia \& Metabologia, 44(1), 81-90.

Margis, R., Picon, P., Cosner, A. F., \& Silveira, R. O. (2003). Relação entre estressores, estresse e ansiedade. Revista de Psiquiatria, 25 (Suplemento 1), 65-74.

Mathias, A., Sanchez, R.P., \& Andrade, M.M.M. (2006). Incentivar hábitos de sono adequados: um desafio para educadores. Núcleos de Ensino, Universidade Estadual Paulista, Editora Unesp, 718-731.

Noal, R. B., Menezes, A. M. B., Canani, S. F., \& Siqueira, F. V. (2008). Ronco habitual e apneia obstrutiva observada em adultos: estudo de base populacional, Pelotas, RS. Revista de Saúde Pública, 42(2), 224-233.

Peppard, P. E., Young, T., Palta, M., \& Skatrud, J. (2000). Prospective study of the association between sleepdisordered breathing and hypertension. The New England Journal of Medicine, 342(19), 1378-1384.

Redmond, G. (1997). Mood disorders in the female patient. International Journal of Fertility and Women's Medicine, 42(2), 67-72.

Sanchez, A. I., Buela-Casal, G., Bermudez, M. P., \& CasasMaldonado, F. (2001). The effects of continuous positive air pressure treatment on anxiety and depression levels in apnea patients. Psychiatry and clinical neurosciences, $55,641-646$.
Saunamäki, T., \& Jehkonen, M. (2007). Depression and anxiety in obstructive sleep apnea syndrome: a review. Acta Neurologica Scandinavica, 116, 277-288.

Schinohara, H., \& Nardi, A. E. (2001). Transtorno de ansiedade generalizada. In: B. Range (Org.). Psicoterapias cognitivo-comportamentais: um diálogo com a psiquiatria (pp. 217-229). Porto Alegre: Artmed.

Schwartz, D. J., \& Karatinos, G. (2007). For individuals with obstructive sleep apnea, institution of CPAP therapy is associated with an amelioration of symptoms of depression which is sustained long term. Journal of Clinical Sleep Medicine, 3(6), 631-635.

Shear, M. K. (1997). Anxiety disorders in women: genderrelated modulation of neurobiology and behavior. Seminars in Reproductive Endocrinology, 15(1), 69-76.

Spielberger, C. D., Biaggio, A., \& Natalicio, L. F. (1979). Manual do Inventário de Ansiedade Traç-Estado. Rio de Janeiro: CEPA.

Stepanski, E. J. (2002). The effect of sleep fragmentation on daytime function. Sleep, 25(3), 268-76.

Young, T., Palta, M., Dempsey, J., Skatrud, J., Weber, S., \& Badr, S. (1993). The occurrence of sleep-disordered breathing among middle-aged adults. The New England Journal of Medicine. 328(17), 1230-1235.

Yue, W., Hao, W., Liu, P., Liu, T., Ni, M., \& Guo, Q. (2003). A case-control study on psychological symptoms in sleep apnea-hypopnea syndrome. Canadian Journal of Psychiatry, 48(5), 318-323.

Nota:

A primeira autora agradece ao $\mathrm{CNPq}$ pela bolsa de estudos concedida. 